\title{
Generation of Tunable Short Pulse VUV Radiation by Four-Wave Mixing in Xenon with Femtosecond KrF-Excimer Laser Pulses
}

\author{
Andreas Tünnermann, Carsten Momma, Kasem Mossavi, Caroline Windolph, and Bernd Wellegehausen
}

\begin{abstract}
A four-wave difference-frequency mixing scheme based on near resonant two-photon excitation of xenon with a femtosecond $\mathrm{KrF}$-excimer laser system is used to generate tunable short pulse radiation in the VUV and UV spectral range between 133 and $355 \mathrm{~nm}$ with output energies of several $\mu \mathrm{J}$ by applying tunable nanosecond laser radiation in the range of $1905 \mathrm{~nm}$ to $185 \mathrm{~nm}$. At the excimer laser wavelengths of 193 $\mathrm{nm}(\mathrm{ArF}), 308 \mathrm{~nm}(\mathrm{XeCl})$ and $351 \mathrm{~nm}(\mathrm{XeF})$, the process has been used to generate high power short pulse radiation by double-pass amplification in an additional excimer laser discharge. So far, output energies of $1.9,3.0$, and $2.8 \mathrm{~mJ}$, respectively, have been obtained at pulse durations in the range of 1 ps. Nonlinear susceptibilities for the difference-frequency mixing process were calculated from a stationary susceptibility formalism and compared to experimental values.
\end{abstract}

\section{INTRODUCTION}

$\mathrm{F}_{\mathrm{s}}$ R the generation of coherent tunable radiation in the VUV spectral range, four-wave mixing is an important and widely used technique. A good review is given by Reintjes [1]. So far, mostly nanosecond pulse laser sources have been used for this process. By applying different gases and vapors tunable radiation from the near VUV down to about $70 \mathrm{~nm}$ has been generated [2]. Up to now, very few experiments have been performed with short pulse radiation [3]-[6].

In two-photon resonant four-wave mixing schemes, which are especially favorable [7], [8], normally two photons of a pump laser field $\left(\omega_{p}\right)$ and one photon of an additional (injected) laser field $\left(\omega_{i}\right)$ are used to generate a signal photon $\left(\omega_{s}\right)$ at either the sum frequency $\omega_{s}=2 \omega_{p}$ $+\omega_{i}$ or the difference frequency $\omega_{s}=2 \omega_{p}-\omega_{i}$. The field with the frequency $\omega_{i}$ may also be internally generated by amplified spontaneous emission (ASE), Raman- or hyperRaman-type processes [9].

Basically, the field $\omega_{s}$ and $\omega_{i}$ may also both develop from quantum noise, if the induced polarization allows a sufficient high gain and phase matching conditions can be accomplished. This process then corresponds to the optical parametric oscillator/amplifier (OPO/OPA) applied in

Manuscript received June 22, 1992; revised September 22, 1992. This work was supported by the Deutsche Forsch-ungsgemeinschaft.

The authors are with the Institut für Quantenoptik. Universität Hannover, Welfengarten 1, 3000 Hannover 1, Germany.

IEEE Log Number 9207484. solids [10] and has been studied in some detail by Wunderlich [11] and by the authors [12].

In principle, the four-wave parametric oscillator (FWPO) scheme is capable of delivering frequencies $\omega_{s}$ and $\omega_{i}$ in a broad spectral range. Due to index matching requirements, however, only distinct frequencies have been observed so far and neither tuning experiments comparable to those of the OPO in crystals have been performed nor broadband radiation has been obtained by this process.

Recently, we have studied nonlinear processes in the two-photon excitation of xenon with a fs-KrF-excimer laser system [12]. We observed intense broad-band emissions in the visible and near infrared spectral range (650$850 \mathrm{~nm})$, coupled with a VUV emission (147-155 nm), and an emission in the UV range $(185-400 \mathrm{~nm})$. The generation process for these emissions was found to be noncollinear phase-matched nonresonant four-wave mixing process starting from quantum noise (FWPO-type process), supported in some cases (visible emission) by internally generated ASE and stimulated hyper-Raman scattering (SHRS) in molecular xenon, serving as input field. In the visible and UV spectral range pulse durations of less than 1 ps have been measured, corresponding to the pump pulse width. The output energies of the emissions exceed several $10 \mu \mathrm{J}$, yielding peak powers in the order of $100 \mathrm{MW}$

In this contribution, four-wave difference-frequency mixing experiments in xenon using a femtosecond $\mathrm{KrF}$ excimer laser $\left(\omega_{p}\right)$ at intensities up to $10^{15} \mathrm{~W} / \mathrm{cm}^{2}$ and tunable nanosecond seed lasers $\left(\omega_{i}\right)$ are described. The process has been used to generate coherent tunable ultrashort pulse radiation in the VUV and UV spectral range $(133-355 \mathrm{~nm})$ with output energies of several $\mu \mathrm{J}$. At the excimer laser wavelengths of $193(\mathrm{ArF}), 308(\mathrm{XeCl})$, and $351 \mathrm{~nm}(\mathrm{XeF})$ the generated radiation was amplified in corresponding excimer laser amplifiers to energies of 1.9 , 3.0 , and $2.8 \mathrm{~mJ}$, respectively.

\section{EXPERIMENTAL}

The experimental arrangement for the generation of tunable short pulse radiation is illustrated schematically in Fig. 1. A fs-KrF-excimer laser system $(248.5 \mathrm{~nm} / 10$ $\mathrm{mJ} / 360 \mathrm{fs}$ ), described in [13] is used to perform a near 


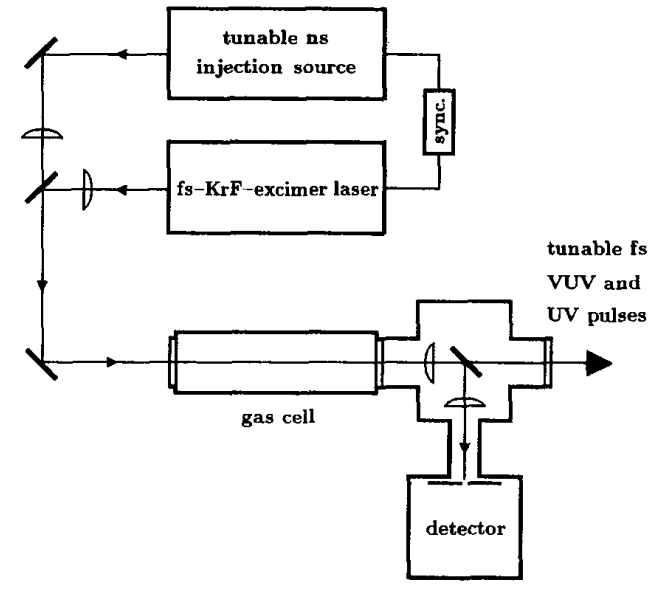

Fig. 1. Experimental arrangement.

resonant two-photon excitation of xenon and induce a strong short-lived nonlinear two-photon polarization (Fig. 2). Different ns laser systems synchronized with the fs laser are applied as injection sources for the differencefrequency mixing process. Either a double discharge excimer laser (Lambda Physik EMG 150 EST) or a Nd: YAG laser (Quanta-Ray Model DCR 2a) have been used as pump sources for different dye lasers. The dye lasers are tunable from 368 to $608 \mathrm{~nm}$, with a spectral width of about $8 \mathrm{GHz}$.

By using a $\mathrm{H}_{2}$-Raman shifter or frequency doubling crystals, the spectral range has been further extended into the UV and near IR. With these pump sources tunable ns radiation in the range of 240 to $1905 \mathrm{~nm}$ can be generated with energies in the $\mathrm{mJ}$ range. It was possible to generate UV pulses down to $185 \mathrm{~nm}$ at specific wavelengths.

One gain module of the excimer laser (amplifier channel) could be operated as an amplifier for the generated short pulse radiation at specific excimer laser wavelengths (see Section IV).

The KrF beam was focused by a $f=1000 \mathrm{~mm}$ lens and the ns beams by a $300 \mathrm{~mm}$ lens into a cell containing xenon at pressures between 1 and 760 torr. The spectral characteristics of the generated UV and VUV radiation were examined with a VUV monochromator (Minuteman 302 VM) and different photomultipliers. Absolute measurements of the generated UV and VUV energy have been performed with a calibrated pyroelectric power meter in combination with spectral filters. The temporal characteristics were studied by a streak camera (a Hadland Imacon 500, with a temporal resolution $\approx 2 \mathrm{ps}$ ) and a Michelson type autocorrelator equipped with an ionization chamber.

\section{Difference Frequency Mixing}

The interaction of xenon with the fs-KrF-excimer laser radiation is dominated by a near resonant two-photon excitation at the $5 p^{6}{ }^{1} S_{0} \rightarrow 6 p[1 / 2]_{0}$ transition (Fig. 2). Relevant single photon resonances for the investigated spec-

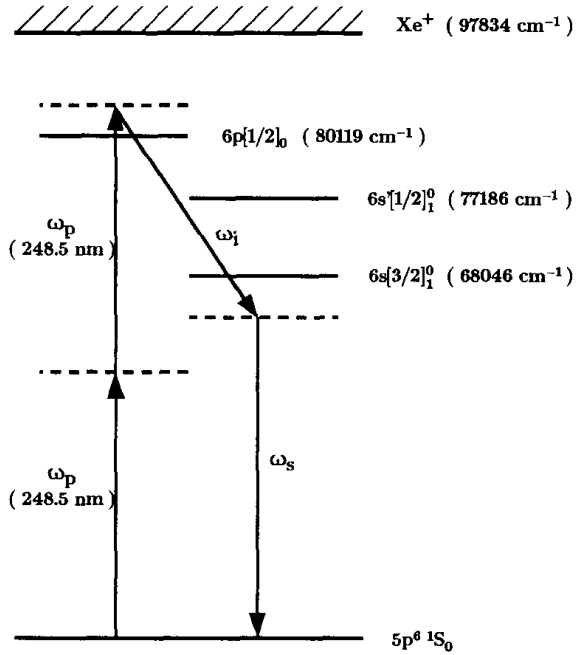

Fig. 2. Relevant energy level scheme of xenon for the four-wave difference mixing process: $\omega_{s}=2 \omega_{p}-\omega_{i}$ with $\lambda_{p}=248.5 \mathrm{~nm}$.

tral range are the $6 s[3 / 2]_{1}^{\circ} \rightarrow 5 p^{6}{ }^{1} S_{0}$ and the $6 s^{\prime}[1 / 2]_{1}^{\circ}$ $\rightarrow 5 p^{6}{ }^{1} S_{0}$ transitions, which prevent the generation of VUV radiation in the vicinity of 147 and $130 \mathrm{~nm}$. Further limitations for the mixing process, especially around 147 and $165 \mathrm{~nm}$, result from an increasing concentration of xenon molecules at higher xenon pressures [14]. Due to the nonresonant excitation process the transient characteristics of the induced two-photon polarization are determined by the fs-KrF-pump laser. In addition, the polarization is strongly damped by the mentioned selfstarting FWPO processes. Therefore, ns pulses can be used as injection pulses in a FWM scheme, where the two-photon polarization is built up by the fs- $\mathrm{KrF}$ laser, to generate ultrashort UV and VUV coherent radiation.

Tunable short-pulse radiation has been generated by four-wave difference-frequency mixing in the spectral range between 133 and $355 \mathrm{~nm}$. Two examples are given in Fig. 3. Fig. 3(a) shows a spectrum obtained from difference FWM of radiation at $\lambda_{i}=1064 \mathrm{~nm}$ and $\lambda_{p}=$ $248.5 \mathrm{~nm}$, which is centered around $141 \mathrm{~nm}$ for an optimum xenon pressure of 80 torr. For a short-pulse energy of $E_{p}=5 \mathrm{~mJ}$ and a Nd:YAG laser energy of $E_{i}=90 \mathrm{~mJ}$, energies of $5 \mu \mathrm{J}$ have been measured. Radiation at $\lambda_{s}=$ $157 \mathrm{~nm}$ with output energies of up to $7 \mu \mathrm{J}$ can be generated in this scheme by using a dye laser at $\lambda_{i}=595 \mathrm{~nm}$ at an energy of $E_{i}=35 \mathrm{~mJ}$ (xenon pressure: 30 torr) as can be seen from Fig. 3(b). Both spectra contain in addition an emission in the wavelength range of 147-154 $\mathrm{nm}$, which is independent of external injection sources. This broad-band emission is due to the self-starting FWPO processes discussed in detail in [12]. The pulse duration of this emission corresponds to the pump laser width due to the off-resonant excitation scheme. In contrast to nsmixing experiments [8], the FWM spectra show for a fixed injection wavelength a remarkable spectral width of approximately $600 \mathrm{~cm}^{-1}$ (FWHM), which is nearly four times larger than the combined bandwidth of two fs-KrF- 


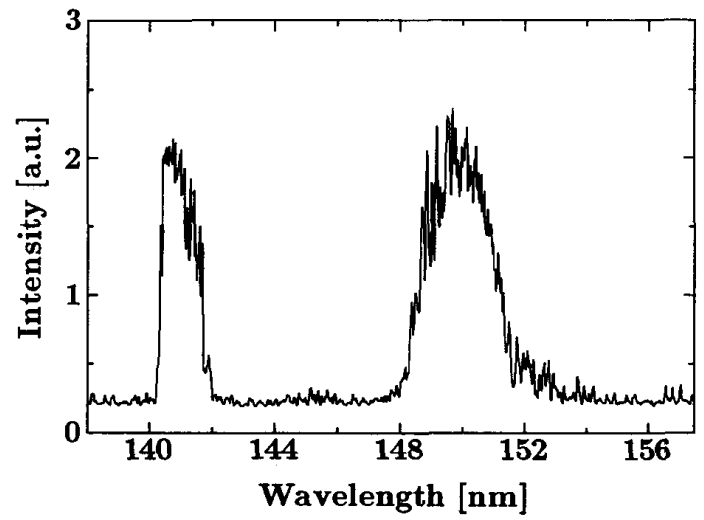

(a)

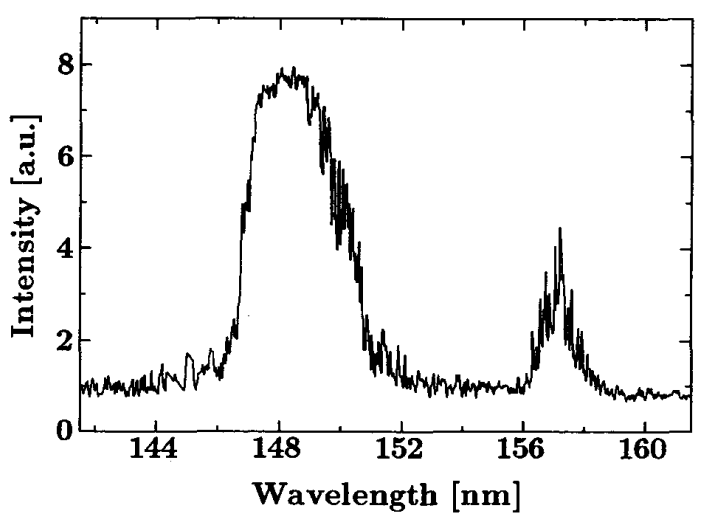

(b)

Fig. 3. Spectra of the four-wave difference mixing process in xenon. The emission around $149 \mathrm{~nm}$ results from four-wave parametric oscillation. (a) Seed pulse: $1064 \mathrm{~nm}$. (b) Seed pulse: $595 \mathrm{~nm}$.

excimer laser photons $\left[160 \mathrm{~cm}^{-1}\right.$ (FWHM)] and the dye laser photon. We assume that the main broadening mechanism is a cascading process due to secondary FWM.

At distinct signal wavelengths (injection wavelengths) parameter dependences have been studied. Fig. 4 gives the output intensity of a generated wave at $\lambda_{s}=193 \mathrm{~nm}$ $\left(\lambda_{i}=352 \mathrm{~nm}\right)$ for different xenon pressures. Optimum conversion efficiency for this process, resulting in output energies of $8 \mu \mathrm{J}$, has been obtained for a xenon pressure of 450 torr, input energies of $10 \mathrm{~mJ}$ for the $\mathrm{fs}-\mathrm{KrF}$-laser radiation and $70 \mathrm{~mJ}$ for the $\mathrm{XeF}$ injection laser. The strong variation of the output energy with the xenon pressure is mainly induced by the phase-matching condition, which is accomplished by a noncollinear interaction with matching angles around $1^{\circ}[12]$. The pulse duration of the generated radiation at $\lambda_{s}=193 \mathrm{~nm}$ was measured by a Michelson type autocorrelator based on two-photon ionization of xenon (xenon ionization potential: $12.13 \mathrm{eV}$ [15], xenon pressure: 20 torr). From the autocorrelation trace (Fig. 5) a pulse duration of 1.0 ps can be calculated assuming a sech ${ }^{2}$ pulse. This pulse duration is determined by a temporal broadening of about $600 \mathrm{fs}$ because of group velocity dispersion effects in the beam separator and the optical components of the autocorrelator. This has been

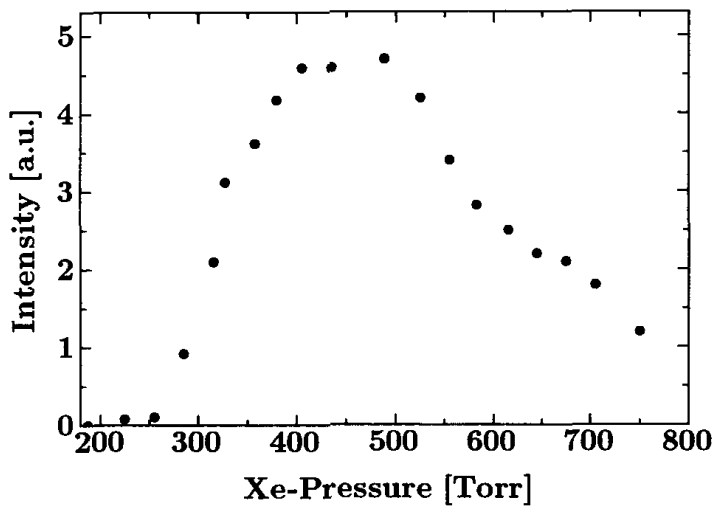

Fig. 4. Output intensity at $193 \mathrm{~nm}\left(\lambda_{i}=352 \mathrm{~nm}\right)$ versus xenon pressure. The maximum corresponds to an energy of about $8 \mu \mathrm{J}$.

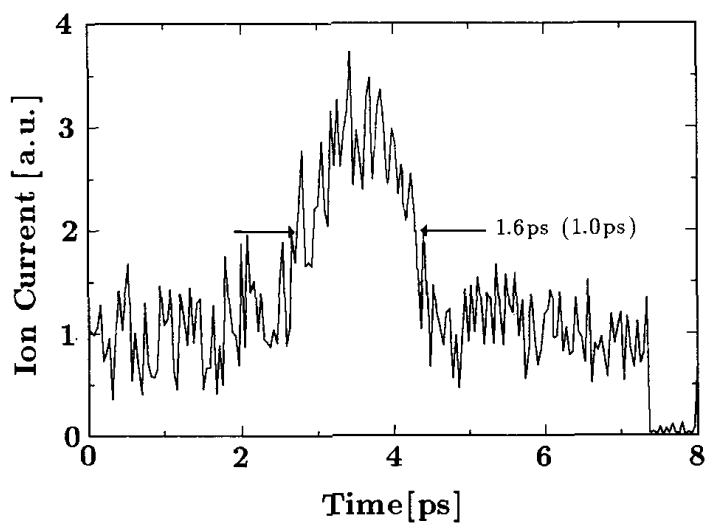

Fig. 5. Autocorrelation trace of the parametric emission at $193 \mathrm{~nm}$.

verified by inserting additional optical materials into the pathway. Comparable pulse durations have also been determined for the reverse process at $\lambda_{s}=352 \mathrm{~nm}\left(\lambda_{i}=\right.$ $193.3 \mathrm{~nm})$. In this case a three-photon ionization process in trimethylamine (TME-ionization potential: $7.82 \mathrm{eV}$ [15], TME-pressure: 10 torr) has been used as nonlinearity in the Michelson-type autocorrelator. Both measurements indicate that the time constants of the mixing process are given by the femtosecond $\mathrm{KrF}$-pump laser pulse as discussed above. By temporal measurements with a streak camera the occurrence of trailing pulses on a picosecond time scale could be excluded.

At various UV and VUV wavelengths between 133 and $355 \mathrm{~nm}$ short pulse radiation with energies in the range of several $\mu \mathrm{J}$ have been measured for input pulses at the $\mathrm{KrF}$ wavelength of 5 to $10 \mathrm{~mJ}$ and seed pulse energies of typically several $10 \mathrm{~mJ}$. The observed conversion efficiencies of about $10^{-2}-10^{-3}$ exceed typical values seen in experiments on third order difference frequency mixing with ns pulses in similar excitation schemes by more than one order of magnitude, which is due to the high peak intensities of the pump laser system. In addition, saturation effects, as the formation of an optically thick plasma by a catastrophic breakdown, which limit the conversion efficiency in ns-experiments, are reduced here. 


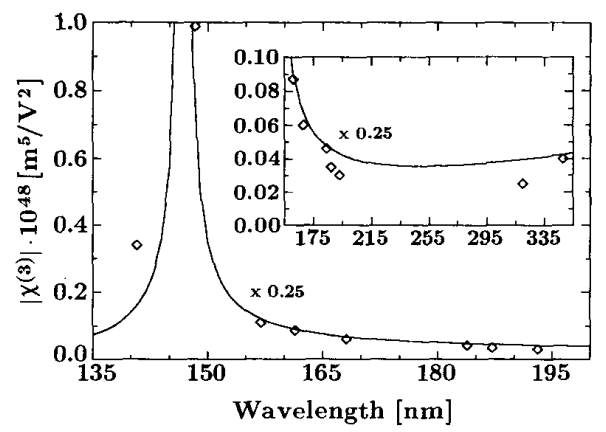

Fig. 6. Nonlinear susceptibility $\left|\chi^{(3)}\right|$ for the difference frequency mixing process in xenon. The rhombes indicates the experimental values.

In the plane wave approximation, which is suited here for estimations of the output power as the interaction length corresponds to the confocal parameter [1] and neglecting absorption effects, the intensity of the radiation generated by FWM is given by [16]

$$
I_{\mathrm{FWM}}=\frac{9 \omega_{s}^{2} N^{2}}{(2 c)^{4} \epsilon_{0}^{2} n_{s} n_{i} n_{p}^{2}}\left|\chi_{\mathrm{FWM}}^{(3)}\right|^{2} l^{2} I_{i} I_{p}^{2} \operatorname{sinc}^{2}\left[\frac{\Delta k l}{2}\right] .
$$

$N$ gives the xenon density, $l$ is the length of the nonlinear medium, and $n_{s}, n_{i}, n_{p}$ are the refraction indexes. For perfect phase matching $\Delta k=2 k_{p}-k_{i}-k_{s}=0$, the generated intensity $I_{\mathrm{FWM}}$ is maximized (sinc $\left.[0]=1\right)$. From the experimental data values of the nonlinear susceptibility for the four-wave mixing process have been determined by (1) and are given by the rhombs in Fig. 6 , assuming an average intensity of $10^{14} \mathrm{~W} / \mathrm{cm}^{2}$ for $I_{p}$. The susceptibility at $\lambda=148.5 \mathrm{~nm}$ and $\lambda=320 \mathrm{~nm}$ has been taken from the FWPO process [12]. The shape of the calculated susceptibility curve (shifted in Fig. 6 by a factor of 0.25 ) agrees well with measured frequency dependence, and also the absolute values fit within one order of magnitude. The calculations are based on a stationary susceptibility formalism [16], assuming a four level system (see Fig. 2), and a fixed fs-KrF-laser detuning of $363 \mathrm{~cm}^{-1}$ from the $6 p[1 / 2]_{0}$ two-photon resonance. Saturation and transient effects, damping rates and also the level shift due to the ponderomotive potential [17] have not been included in the calculations. Necessary atomic data of oscillator strengths were taken from [18].

\section{Amplification Experiments}

By means of the four-wave difference frequency mixing process in xenon, tunable short-pulse radiation in the VUV and UV spectral range with output powers in the MW range can be generated. As essential increase of the output power at specific wavelengths can be realized by further amplification. Established active media for the amplification of picosecond and subpicosecond pulses in the UV spectral range are rare gas halide excimers, because of their high gain coefficients and gain bandwidth [19]. For a sufficient amplification of fs-pulses, injection pulses of an energy density in the range of several $\mu \mathrm{J} / \mathrm{cm}^{2}$ are necessary to drive the gain medium into saturation.
TABLE I

Amplification of Femtosecond Pulses in Excimer Gain Modules

\begin{tabular}{cccc}
\hline $\begin{array}{c}\text { Amplifier } \\
\text { Wavelength } \\
{[\mathrm{nm}]}\end{array}$ & $\begin{array}{c}\text { Input Pulse }{ }^{(\mathrm{a})} \\
\text { Generation Process }\end{array}$ & Output Energy [mJ] & (Single Pass) \\
\cline { 3 - 4 } $\begin{array}{c}349,351,353 \\
(\mathrm{XeF})^{1}\end{array}$ & $\begin{array}{c}\text { FWM } \\
\left(\lambda_{i}=193 \mathrm{~nm}\right)\end{array}$ & 0.26 & 2.8 \\
$\begin{array}{c}308 \\
(\mathrm{XeCl})^{2}\end{array}$ & FWPO & 0.50 & 3.0 \\
$\begin{array}{c}193 \\
(\mathrm{ArF})^{3}\end{array}$ & $\begin{array}{c}\text { FWM } \\
\left(\lambda_{i}=351 \mathrm{~nm}\right)\end{array}$ & 0.26 & 1.9 \\
\hline
\end{tabular}

(a) Subpicosecond pulse with energy density typically $>5 \mu \mathrm{J} / \mathrm{cm}^{2}$

${ }^{\text {(b) }}$ Pulse duration in the range of $1 \mathrm{ps}$

'XeF-gas mixture: $\mathrm{Xe}$ : 15 torr, $\mathrm{He} / \mathrm{F}_{2}$ : 165 torr, $\mathrm{He}$ : 1770 torr

${ }^{2} \mathrm{XeCl}$-gas mixture: $\mathrm{Xe:} 75$ torr, $\mathrm{He} / \mathrm{HCl}$ : 60 torr, He: 1740 torr

${ }^{3}$ ArF-gas mixture: Ar: 260 torr, $\mathrm{He} / \mathrm{F}_{2}: 115$ torr, $\mathrm{He}: 975$ torr

At the excimer wavelengths of 193 (ArF), $308(\mathrm{XeCl})$, and $351 \mathrm{~nm}(\mathrm{XeF})$ we have performed amplification experiments using the amplifier channel of the double discharge excimer laser, whereas the oscillator channel was used to generate the seed pulses for the FWM process. The slightly divergent UV injection pulses with energies in the $\mu \mathrm{J}$ range pass through the amplifier channel of the excimer laser, which is filled with usual ArF-, $\mathrm{XeCl}-$, or XeF-mixtures, respectively (see Table I). After the first pass, the beam is spatially filtered by an evacuable pinhole to reduce the ASE fraction and magnified by a beam expanding telescope ( $f=140 \mathrm{~mm}$ and $f=50 \mathrm{~mm}$ ) to fill the whole aperture of the amplifier (cross section $28 \mathrm{~mm}$ $\times 8 \mathrm{~mm}$ ) in the second pass.

For the amplification of the $308 \mathrm{~nm}$ radiation, the UV continuum generated directly by the self-starting FWPO process has been used [12]. The spectral characteristic of this input signal is displayed in Fig. 7(a). For the total spectral range an energy of about $70 \mu \mathrm{J}$ has been measured [12]. After double pass amplification the spectrum of the UV pulse [Fig. 7(b)] is narrowed according to the gain profile of $\mathrm{XeCl}$. Output energies up to $3 \mathrm{~mJ}$ have been measured.

For the amplification of seed pulses around $351 \mathrm{~nm}$ in XeF [pulse energy: $20 \mu \mathrm{J}$; Fig. 8(a)] an output energy of $2.8 \mathrm{~mJ}$ has been observed. After double-pass amplification the spectrum of this pulse consists of three lines centered around 349,351 , and $353 \mathrm{~nm}$ [Fig. 8(b)]. In comparison to the reported ASE spectrum in [20], where only two lines at 351 and $353 \mathrm{~nm}$ appear, the seeding of the XeF amplifier with the FWM signal [Fig. 8(a)] leads to the observation of a third line, indicating a nonzero gain a $349 \mathrm{~nm}$ [Fig. 8(b)]. Considering potential curves [21] and Franck-Condon factors [22], this line can be attributed to a $\mathrm{XeF}\left(B, v^{\prime}=2\right) \rightarrow \mathrm{XeF}\left(X, v^{\prime \prime}=5\right)$ transition.

For amplification in the ArF gain module, the corresponding input and output spectra are given in Fig. 9(a) and (b). For seed pulses of about $8 \mu \mathrm{J}$, output energies of so far $1.9 \mathrm{~mJ}$ have been measured at a pulse duration of $1.4 \mathrm{ps}$. Therefore this source is one of the most intense lasers at the vacuum UV cutoff [6], [23], [24]. Due to the high input energies a saturation of the ArF amplifier for 


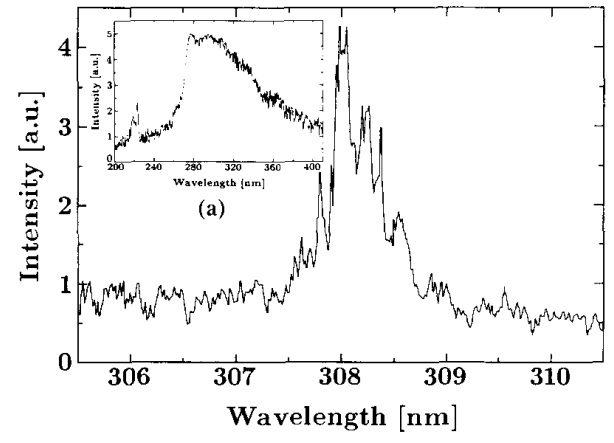

(b)

Fig. 7. Amplification of subpicosecond pulses at the $\mathrm{XeCl}$ wavelength. (a) Spectrum of the input pulse, generated by four-wave parametric oscillation. (b) Spectrum of the amplified short pulse.

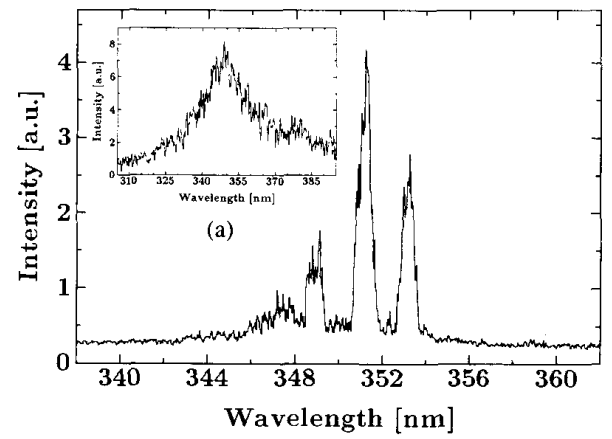

(b)

Fig. 8. Amplification of subpicosecond pulses at the $\mathrm{XeF}$ wavelength. (a) Spectrum of the input pulse, generated by four-wave difference frequency mixing $\left(\lambda_{i}=193 \mathrm{~nm}\right)$. (b) Spectrum of the amplified short pulse.

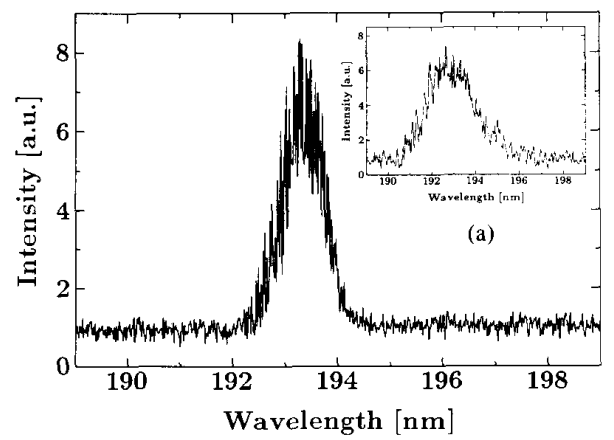

(b)

Fig. 9. Amplification of subpicosecond pulses at the ArF wavelength (a) Spectrum of the input pulse, generated by four wave difference frequency mixing $\left(\lambda_{i}=352 \mathrm{~nm}\right)$. (b) Spectrum of the amplified short pulse.

subpicosecond seed pulses has been observed for the first time. Investigations of the small-signal gain coefficient, the saturation energy density and the transient amplification characteristics for ultrashort pulses in an ArF gain module are in progress [25]. Specific data for single- and double-pass amplification for the different wavelengths are summarized in Table I. Typically, a slight temporal broadening of the amplified pulse in the range of $25 \%$ is observed compared to the injected pulse. The ASE energy component on a ns time scale after the second pass was typically in the order of $10 \%$.

\section{Conclusion}

Nonresonant four-wave mixing with high intensity short pulse and short-wavelength pump sources seems to be a promising technique for the generation of intense tunable short-pulse radiation in the UV and VUV. Comparable schemes as discussed here for xenon should be possible in other atomic or molecular gases such as $\mathrm{Hg}$ or $\mathrm{CO}$. Starting from intense pulses at $193 \mathrm{~nm}$ (ArF) sum- and difference-frequency mixing processes in $\mathrm{Kr}, \mathrm{H}_{2}$, or $\mathrm{Ne}$ should lead to the generation of fs pulses below $100 \mathrm{~nm}$. With the generation of fs seed pulses in the VUV, amplification in other short-wavelength gain media such as $F_{2}$ $(157 \mathrm{~nm}), \mathrm{Xe}_{2}(172 \mathrm{~nm}), \mathrm{Kr}_{2}(146 \mathrm{~nm})$, and $\mathrm{Ar}_{2}(126 \mathrm{~nm})$ appears feasible, with the potential to generate high peak power pulses at these wavelengths. Such sources might then open up new possibilities for the generation of deep VUV and even soft X-rays by low order nonlinear processes.

Note Added in Proof: In first experiments with a more powerful excimer laser (Lambda Physik LPX 250), having a longer amplification channel, output energies up to $8 \mathrm{~mJ}$ at $193 \mathrm{~nm}$ have been measured.

\section{REFERENCES}

[1] J. F. Reintjes, Nonlinear optical parametric processes in liquids and gases. London: Academic, 1984

[2] R. C. Elton, X-Ray Lasers. London: Academic, 1990.

[3] A. H. Kung, "Generation of tunable picosecond VUV radiation," Appl. Phys. Lett., vol. 25, pp. 653-654, 1974.

[4] N. P. Economou, R. R. Freeman, J P. Heritage, and P. F. Liao, "Vacuum ultraviolet picosecond pulse generation using $\mathrm{cw}$ modelocked dye lasers,"' Appl. Phys. Lett., vol. 36, pp. 21-23, 1980.

[5] T. R. Royt and Chi H. Lee, "Spectroscopic studies in the picosecond domain with synchronously mode-locked dye lasers." Appl. Phys. Lett., vol. 30, pp. 332-335, 1977.

[6] J. H. Glownia, M. Kaschke, and P. P. Sorokin,, “Amplification of $193 \mathrm{~nm}$ femtosecond seed pulses generated by third-order nonresonant, difference-frequency mixing in xenon, "Opt. Lett., vol. 17, pp. 337-339, 1992.

[7] S. E. Harris and R. B. Miles, "Proposed third-harmonic generation in phase-matched metal vapors," Appl. Phys. Lett., vol. 19, pp. 385387,1971 .

[8] R. Hilbig and R. Wallenstein, "Tunable VUV radiation generated by two-photon resonant frequency mixing in xenon," IEEE J. Quantum Electron., vol. QE-19, pp. 194-201, 1983.

[9] J. Bokor, R. R. Freeman, R. L. Panock, and J. C. White, "Generation of high-brightness coherent radiation in the vacuum ultraviolet by four-wave parametric oscillation in mercury vapor," Opt. Lett., vol. 6 , pp. 182-184, 1981 .

[10] P. G. Harper, and B. S. Wherrett Nonlinear Optics. London: Academic, 1977

[11] R. K. Wunderlich, W. R. Garrett, R. C. Hart, M. A. Moore, and M. G. Payne, "Nonlinear optical processes near the sodium 4D two-photon resonance," Phys. Rev. A, vol, 4I, pp. 6345-6360, 1990.

[12] A. Tünnermann, K. Mossavi, and B. Wellegehausen, "Nonlinear optical processes in the near resonant two-photon excitation of xenon by femtosecond KrF-excimer laser pulses," Phys. Rev. A, vol. 46. p. 2707,1992

[13] S. Szatmari and F. P. Schäfer, "Simplified laser system for the gen eration of $60 \mathrm{fs}$ pulses at $248 \mathrm{~nm}$, , Opt. Commun., vol. 68, pp. 196202,1988 
[14] M. C.. Castex, "Experimental determination of the lowest excited $\mathrm{Xe}_{2}$ molecular states from VUV absorption spectra," J. Chem. Phys. vol. 74, pp. 759-771, 1981

[15] J. A. R. Samson, Techniques of Vacuum Ultraviolet Spectroscopy New York: Wiley, 1967

[16] D. C. Hanna, M. A. Yuratich, and D. Cotter, Nonlinear Optics of Free Atoms and Molecules. Berlin: Springer Verlag, 1979.

[17] M. Dörr, R. M. Potvliege, and R. Shakeshaft, "Multiphoton processes in an intense laser field. III: Resonant ionization of hydrogen by subpicosecond pulses,"'Phys. Rev. A, vol. 41, pp. 558-561, 1990.

[18] M. Aymar and M. Coulombe, "Theoretical transition probabilities and lifetimes in Kr I and Xe I spectra," Atomic Data and Nuclear Data Tables, vol. 21, pp. 537-566, 1978.

[19] P. H. Bucksbaum, J. Boker, R. H. Storz, and J. C. White, "Amplification of ultrashort pulses in krypton fluoride at $248 \mathrm{~nm}$," Opt. Lett., vol. 7, pp. 399-401, 1982

[20] Q. Zhao, S. Szatmari, and F. P. Schäfer, "Gain dynamics of XeF and subpicosecond pulse generation at $351 \mathrm{~nm}$," Appl. Phys. B, vol. 47, pp. 325-332, 1988.

[21] S. F. Fulghum, M. S. Feld, and A. Javan, "A multilevel model of XeF ground state kinetics," IEEE J. Quantum Electron., vol. QE-16, pp. 815-820, 1980.

[22] P. C. Tellinghuisen, J. Tellinghuisen, J. A. Coxon, J. E. Velazco, D. W. Setser, "Spectroscopic studies of diatomic noble gas halides. IV: Vibrational and rotational constants for the X, B, and D states of XeF," J. Chem. Phys., vol. 68, pp. 5187-5198, 1978.

[23] H. Egger, T. S. Luk, K. Boyer, D. F. Muller, H. Pummer, and T. Srinivasan, "Picosecond, tunable ArF* excimer laser source," Appl. Phys. Lett., vol. 41, pp. 1032-1034, 1982.

[24] S. Szatmari and F. P. Schäfer, "Generation of input signals for ArF amplifiers," J. Opt. Soc. Amer. B, vol. 6, pp. 1877-1883, 1989.

[25] C. Momma, H. Eichmann, H. Jacobs, A. Tünnerman, B. Wellegehausen, "Short pulse amplification and gain dynamics of the ArFexcimer," Opt. Lett., vol. 18, Apr. 1993.

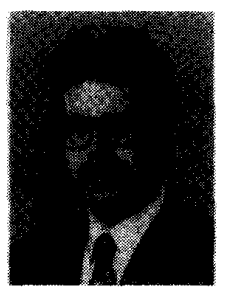

Andreas Tünnermann was born in Ahnsen, Germany, on June 10,1963 . He received the M.S. and $\mathrm{Ph} . \mathrm{D}$. degrees in physics from the University of Hannover in 1988 and 1992, respectively.

$\mathrm{He}$ is currently a member of the Institut für Quantenoptik, University of Hannover. His present research activities are in the field of nonlinear processes with emphasis on the interaction of high intensity laser sources with matter.

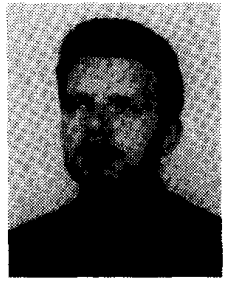

Carsten Momma was born in Barsinghausen, Germany, on March 16, 1963. He received the M.S. degree in physics from the University of Hannover in 1990.

$\mathrm{He}$ is presently working towards the Ph.D. degree in physics at the University of Hannover. His research interests include the development of coherent short pulse sources in the VUV.

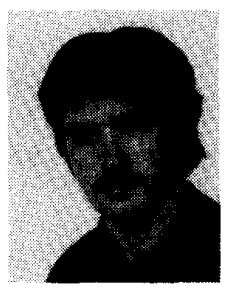

Kasem Mossavi was born in Hannover, Germany, on December 4, 1964. He received the M.S. degree in physics from the University of Hannover in 1991.

Since 1992 he has been a visiting scholar with the Department of Electrical and Computer Engineering, Rice University, Houston, TX. His main research interests include scientific and technical aspects associated with short pulse laser physics and the generation of coherent short wavelength radiation.

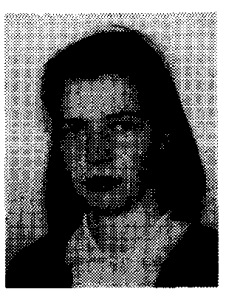

Caroline Windolph was born in Walsrode, Germany, in 1968.

Presently, she is working towards the M.S. degree in physics at the University of Hannover.

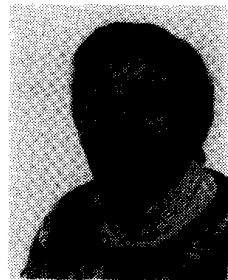

Bernd Wellegehausen was born in Hannover Germany, on August 25, 1942. He received the M.S. and $\mathrm{Ph}$.D. degrees in physics from the University of Hannover in 1968 and 1972, respectively.

Since 1985 he has been a professor with the Institut für Quantenoptik, University of Hannover, and leads a research group focussing on the development of short wavelength lasers. His special research interests are innershell photoionization lasers, anit-Stokes Raman lasers, and nonlinear optical processes. 